\title{
Dynamic control and quantification of bacterial population dynamics in droplets
}

\author{
Shuqiang Huang ${ }^{\text {a }}$, Jaydeep K Srimani ${ }^{\text {a }}$, Anna J Lee ${ }^{\text {a }}$, Ying Zhang ${ }^{\text {a, c }}$, Allison J Lopatkin ${ }^{\text {a }}$, Kam \\ $\mathrm{W}^{\mathrm{Leong}}{ }^{\mathrm{c}}$ and Lingchong You ${ }^{\mathrm{a}, \mathrm{b}}{ }^{\mathrm{b}}$ \\ ${ }^{a}$ Department of Biomedical Engineering, Duke University, Durham, NC, USA \\ ${ }^{\mathrm{b}}$ Duke Center for Genomic and Computational Biology, Duke University, Durham, NC, USA \\ ${ }^{\mathrm{c}}$ Department of Biomedical Engineering, Columbia University, New York, USA \\ * Corresponding author. CIEMAS 2355, 101 Science Drive, Box 3382, Durham, NC 27708, \\ USA. Tel.: +1 919660 8408; Fax: +1 919668 0795; E-mail: you@ duke.edu
}

\begin{abstract}
Culturing and measuring bacterial population dynamics are critical to develop insights into gene regulation or bacterial physiology. Traditional methods, based on bulk culture to obtain such quantification, have the limitations of higher cost/volume of reagents, non-amendable to small size of population and more laborious manipulation. To this end, droplet-based microfluidics represents a promising alternative that is cost-effective and high-throughput. However, difficulties in manipulating the droplet environment and monitoring encapsulated bacterial population for long-term experiments limit its utilization. To overcome these limitations, we used an electrode-free injection technology to modulate the chemical environment in droplets. This ability is critical for precise control of bacterial dynamics in droplets. Moreover, we developed a trapping device for long-term monitoring of population dynamics in individual droplets for at least 240 hours. We demonstrated the utility of this new microfluidic system by quantifying population dynamics of natural and engineered bacteria. Our approach can further improve the analysis for systems and synthetic biology in terms of manipulability and high temporal resolution.
\end{abstract}

Key words: Dynamic droplet manipulation; Bacterial population dynamics; Droplet injection; Microfluidics 


\section{Introduction}

Quantification of bacterial population dynamics and gene expression is critical for developing quantitative insights into gene regulation or bacterial physiology [1-5]. Examples include quantifying bacterial responses to diverse environmental cues or characterizing dynamics of engineered circuits $[6,7]$. Measurements of bacterial populations are often carried out in bulk culture $(100 \mu \mathrm{L}-30 \mathrm{~mL})$, using culture tubes or plate readers. These approaches are not costeffective, in particular, when a large number of measurements are needed or when precious samples or reagents are involved. Moreover, they are not readily amenable to analyzing dynamics of extremely small populations. Yet, quantification of such dynamics is critical to understand the evolution of cooperation, where the extent of heterogeneity in the population structure can dictate the evolutionary outcomes [8-10]. Recent studies have relied on extremely strong dilutions to generate such heterogeneity in different wells of a microplate [10-14]. This strategy, however, involves laborious manipulation of bacterial cultures, tedious splitting and mixing processes, and is prone to experimental errors (e.g. repetitive, manual pipetting).

Droplet-based microfluidics represents a promising alternative that can overcome these technical limitations. Generation of droplets relies on the emulsion of water in oil or vice versa. A single round of emulsion generates water-in-oil droplets [15]; two rounds of emulsion generates waterin-oil-in-water droplets (or double emulsion droplets) [16]. In each case, the water phase can be used for culturing cells. That is, each droplet can be used as a miniaturized reactor that allows culturing and quantification of cell populations. The droplets can be produced at a high speed $(\sim 5300$ droplets per second [17]), and with a narrow size distribution $(<3 \%$ in standard deviation of size distribution [18]). As these droplets can be made extremely small (potentially $1 \mathrm{pL}$ per droplet or even smaller [17]), the cost of reagents for their production is much lower than typical bulk-culture experiments. More importantly, their small sizes can also facilitate generation of a 
large number of small bacterial populations with varying degree of heterogeneity in population distribution for evolutionary studies.

Thanks to these advantages, droplets have been adopted for the analysis of gene expression in bacterial populations [19-23], and for screening drug responses of mammalian cells [24]. To date, however, application of the droplet technology is limited by the lack of manipulability of the chemical environment in the droplets, and the limited capability of long-term monitoring to acquire high temporal resolution required for such studies.

To address these limitations, we have developed a microfluidic system that is integrated with an electrode-free injection technique [25] for dynamic manipulation of droplets, in terms of their volume and composition. We further developed a trapping device to allow long-term, stable maintenance and monitoring of individual droplets, which also enables acquisition of growth dynamics with high temporal resolution. As a demonstration of our platform, we used this microfluidic system to characterize both natural bacteria and engineered bacteria in response to diverse environmental cues, such as cell density and antibiotics. In each case, population dynamics in the droplets are consistent with previous measurements in bulk cultures. Our results highlight the advantages of using droplet technology for versatile manipulation and monitoring of small bacterial populations. 


\section{Results}

\section{Droplet injection and long-term data acquisition}

Our microfluidic device was fabricated using polydimethylsiloxane (PDMS) [26]. It consists of a droplet-production unit, an injecting unit, and a mixing unit (Figure 1A, Supporting information). To produce droplets, we used fluorocarbon oil Novec $^{\mathrm{TM}} 7500$ containing $1 \%$ surfactant (Pico-Surf ${ }^{\mathrm{TM}} 1$, Sphere Fluidics) as the oil phase, and M9 medium with or without bacteria as the water phase. Two syringe pumps were used to control the flow rate of each phase, which then determined the production rate of droplets. To manipulate the environment in droplets, we used an electrode-free injection technique to deliver chemicals into the droplets after they were generated. A DC power source (3B Scientific U33010-115) was used to trigger injection (Figure 1A).

When the power source is OFF, the droplet is stabilized by surfactant molecules: the hydrophilic head of the surfactant is embedded into the droplet while the hydrophobic tail remains in the outer oil phase, forming a stable layer surrounding the droplet [27]. When the droplet moves across the orifice of injection phase, injection does not occur due to this chemical stability. Instead, the injection channel generates separate droplets that move along with the original droplets (Figure 1B). However, when the power source is $\mathrm{ON}$, the electric field destabilizes the surfactant layer of each droplet as it passes the orifice. The injection channel can then deliver a small amount of liquid into the droplet, forming a larger one (Figure 1B, Supporting Video S1). The mixing unit downstream is designed to mix the injection phase and bacterial cells within droplets as they flow through the winding microstructure [28].

To study the effects of electric field on cell survival, we compared cell growth in droplets that were generated with and without droplet injection. We produced droplets containing low density of bacterial cells (MC4100 with a GFP reporter construct) and then injected M9 medium into 
these droplets at varying voltages (up to $5 \mathrm{KV}$ ). The injected droplets were trapped and cultured at $37^{\circ} \mathrm{C}$. The mean intensity of GFP was used as readout of cell density in individual droplets. Compared with the control droplets without injection $(0 \mathrm{KV})$, no significant difference in growth kinetics was observed within the range of voltages that we used, which suggests that the effects of electric field were negligible (Figure 1C).

A distinct feature of our technique is its ability to precisely control the property of droplets in terms of injection efficiency (the percentage of droplets that are successfully injected), the dilution ratio of injected chemicals (DR, final concentration of chemicals in injected droplet versus initial concentration in injection phase), and the homogeneity of droplet size. Depending on specific applications, one may adjust the operating parameters to modulate the properties of the droplets accordingly.

In particular, the droplet generation rate and the applied voltage had opposing effects on the injection efficiency. The injection efficiency decreased with the droplet production rate (Figure 2A) but increased with the applied voltage (Figure 2B). At a high production rate, the fast moving speed of droplets did not provide sufficient time for the re-arrangement of surfactant molecules and the contact between droplet and injection phase. In contrast, a higher voltage generated a stronger electric field that would more effectively destabilize the surfactant layer. Thus, a high production rate of droplets would require a higher voltage to maintain the high injection efficiency (Figure 2C). When the voltage was too high, however, it could result in unstable flow, leading to heterogeneity in droplet size. In our subsequent experiments, we used a flow rate of $1.0 \mu \mathrm{l} / \mathrm{min}$ for both water and oil phases and a voltage of $3 \mathrm{KV}$ to balance the throughput of droplet generation, the injection efficiency, and the homogeneity of injected droplets. This voltage did not cause appreciable variability in cell survival (Figure 1C). 
We then used another syringe pump to control the flow rate of injection phase, and in turn to control DR of the injected chemicals. We found that the DR increased with the flow rate of injection phase (Figure S1, Supporting Information). However, a high flow rate of the injection phase caused more heterogeneity in droplet size and composition: under this condition, the injection phase would fail to inject into some crossing droplets and instead form empty droplets. In our experiments, we use a flow rate of $0.1 \mu 1 / \mathrm{min}$ for the injection phase to obtain monodisperse droplets, while maintaining a sufficiently high DR.

Acquisition of long-term chemical or biological dynamics in individual droplets has been challenging. Measurements in past studies $[29,30]$ were typically obtained by manually loading and unloading droplets at specific time points, or by focusing on the global population of droplets $[16,24]$. The challenge is primarily due to the motility of and lack of long-term stability of droplets. The instability of droplets is often caused by the evaporation of fluorocarbon oil and water in droplets through the permeable structure of PDMS polymer and reservoirs, especially for long-term culturing at a high temperature. When evaporation occurs, the droplets become unstable and will eventually collapse without the support of the outer oil phase. Herein, we introduced a method to improve the droplet stability and to reduce their motility. We first fabricated a microfluidic device with multiple trapping regions to confine the droplets (Figure S2). We then sealed it with Epoxy glue (LOCTITE, heavy duty) to the bottom of a $60 \mathrm{~mm}$ petri dish that was precut to the size of the chip. After the droplets were loaded, $10 \mathrm{~mL}$ water was poured into the petri dish to cover the trapping device. Immersion in water allows maintenance of humidity in the device and prevents evaporation of the oil and droplets. Finally, we used parafilm to seal the petri dish (Figure S3A). Using this method, the droplets could be maintained and monitored for at least 240 hours, which indicated a high level of stability (Figure S3B).

\section{Quantitative analysis of bacterial dynamics in droplets}


To demonstrate the capability of this droplet technology, we used it to quantify different population dynamics generated by either engineered or natural bacterial strains. To simplify the interpretation of our experimental results, we chose well-characterized bacterial strains, which allowed us to evaluate the consistency between experiments in droplets and those in bulk cultures.

\section{Programmed population control by an ePop circuit}

We first used the droplet platform to quantify the population dynamics of engineered E. coli MC4100Z1 carrying the ePop circuit [31], which encodes a lysis gene (E) from phage $\phi X 174$ [32,

33] (Figure 3A inset). Specifically, the plasmid copy number in each cell increases with the population density, leading to an increased basal level production of E protein. Accumulation of E protein results in the death of a subpopulation by inhibiting cell wall synthesis. The remaining population recovers until the density is high enough to induce another round of lysis. Under appropriate experimental conditions, this density-dependent lysis can cause population-level oscillations $^{21}$.

When ePop cells were cultured in droplets, they generated population dynamics consistent with the circuit function [31] (Figure 3A). Both microscopy images and quantification of mean GFP intensity of individual droplets indicated the population-level collapse and recovery. The population in each droplet started from a low density (1 5 cells per droplet), and reached its threshold density at approximately the 6th hour when the population crashed. The population then recovered at roughly the $20^{\text {th }}$ hour. All population dynamics from sampled droplets exhibited qualitatively similar trends but with observable variability. This variability was likely due to heterogeneity of engineered cells (e.g. variable E gene expression) and initial cell density in droplets.

\section{Inoculum effect in response to antibiotics}


The high throughput nature of droplet technology also lends itself to rapid screening analysis, e.g. dose response in antibiotic treatment. To this end, we investigated the inoculum effect (IE) of a lab bacterial strain. IE refers to a population-dependent phenomenon in which bacteria at high initial densities are able to survive with intermediate antibiotic concentrations, while populations at low initial densities are eliminated [34-36]. IE has been observed in response to multiple antibiotics in a clinical setting, and can result in increased mortality [37].

Density-dependent survival due to antibiotics can arise via a number of mechanisms[36]; here, we focus on population-wide antibiotic titration coupled with ribosome inhibition using a known IEgenerating antibiotic, streptomycin (Strep). As shown in Figure 3B, ribosomes (Rs) accumulate in the cytoplasm as a result of transcription and translation, yielding a positive feedback loop [35]. Extracellular Strep (A) is allocated to each cell in accordance with influx and efflux rates, and binds to ribosomes (RsA), leading to inhibition of protein synthesis and ultimately cell death [38]. Strep can also cause heat shock response (HSR) due to translational errors [39, 40], which will further degrade the ribosomal RNA and proteins [41]. HSR is also critical for generation of IE by this mechanism [35]: for an intermediate antibiotic concentration, a population with a high initial density can survive but one with a low initial density cannot. Outside this range, both initial conditions lead to growth at sufficiently low antibiotic concentrations or death at sufficiently high antibiotic concentrations (Figure 3B right).

To quantify IE in droplets, we transformed the sensitive bacterial strain BW25113 with a GFP reporter (pZS31GFP). Overnight culture of engineered bacteria was diluted by 10 folds and recultured in $\mathrm{M} 9$ for 2 hours $\left(\mathrm{OD}_{600} \sim 0.2\right)$. We then diluted the resulting culture by 10 folds again in fresh M9 medium to generate the high-initial density culture, or by 100 folds to generate the lowinitial density culture. Both were supplemented with appropriate concentrations (from $1 \mu \mathrm{g} / \mathrm{ml}$ to $10 \mu \mathrm{g} / \mathrm{ml}$ ) of Strep, before being used as water phase to produce droplets. 
When Strep was below $4 \mu \mathrm{g} / \mathrm{ml}$, droplets with both high and low initial cell densities grew to a high final density. When Strep was above $4 \mu \mathrm{g} / \mathrm{ml}$, neither of them could grow (Figure 3D top row, Figure S4). Importantly, when Strep was $4 \mu \mathrm{g} / \mathrm{ml}$, the droplets with a high initial density survived, while those with a low initial density did not. This density-dependent survival is the defining character of IE. In contrast, antibiotics that do not induce HSR and rapid ribosome degradation will not cause IE [35]. Here, we used chloramphenicol $(\mathrm{Cm})$ as an example (Figure 3D bottom row, Figure S4). Droplets with both high and low cell densities either grew $([\mathrm{Cm}] \leq$ $4 \mu \mathrm{g} / \mathrm{ml})$ or were inhibited $([\mathrm{Cm}]>4 \mu \mathrm{g} / \mathrm{ml})$.

\section{Programmed altruistic death of engineered bacteria in droplets}

We then examined the dynamics of a synthetic gene circuit that programs altruistic death (PAD) in bacteria. Our lab recently developed this circuit to analyze evolutionary dynamics of programmed death in bacteria [14] (Figure 4A left). The circuit contains a lysis module and a public-good module. The lysis module consists of the E gene from phage $\phi \mathrm{X} 174$, as in the ePop circuit (Figure 3A), and is under control of the $\mathrm{P}_{\mathrm{ampc}}$ promoter. The public-good module consists of an isopropyl $\beta$-D-1-thiogalactopyranoside (IPTG) inducible beta-lactamase (BlaM). BlaM is modified from a wild-type Bla such that it is cytoplasmic [11].

A normally non-lytic antibiotic, (+)-6-aminopenicillanic acid (6-APA), can cause partial cell wall breakdown, which generates a small molecule (anhMurNAc-tripeptide). This small molecule induces expression of $\mathrm{E}$ protein from the $\mathrm{P}_{\mathrm{ampC}}$ promoter $[14,42]$. When this occurs, a subpopulation of cells will be lysed, releasing BlaM into the culture. At a high enough concentration, BlaM can degrade the antibiotic and allow the remaining population to survive. As a control, we used a circuit without the lysis module, termed non-programmed death (NPD) (Figure 4A right). In order to quantify population dynamics, GFP and mCherry were placed 
under the constitutively expressed promoter $\mathrm{P}_{\text {tet }}$ for PAD and NPD, respectively. We chose these two circuits in part due to their non-trivial dynamics in response to varying antibiotic concentrations and in part to demonstrate the capability of droplet injection technique.

Droplets containing the PAD strain $\left(\mathrm{OD}_{600} \sim 0.1\right)$ flowed across the orifice of the injection phase, and a high concentration of 6-APA was injected into these droplets. The final concentration of 6APA in the droplets was calculated to be approximately $400 \mu \mathrm{g} / \mathrm{ml}$ (Figure S1, Supporting Information), which proved inhibitory to the cell populations (shown as PAD + 6-APA in Figure 4B and Figure 4C). When the same volume of M9 medium was injected by applying the same parameters, the droplets grew to a high density (PAD - 6-APA), indicating that cell death was indeed due to 6-APA. In contrast, NPD cells were much more tolerant to the same concentration of 6-APA (as NPD +6-APA) compared with those without 6-APA treatment (as NPD - 6-APA), due to the lack of E lysis module.

The survival and death of PAD cells is tunable by two chemicals, IPTG and 6-APA. This property makes the circuit an excellent test-bed for the injection technique. Here, we modulated the final concentrations of 6-APA and IPTG in droplets by varying their concentrations in the injection phase. At $25 \mu \mathrm{g} / \mathrm{ml} 6$-APA, the population without any induction of BlaM production initiated growth for the first 510min but stopped growing thereafter (green line in Figure 5A). In comparison, when the population was induced by $1 \mathrm{mM}$ IPTG, the cells grew to a higher density; this is due to the degradation of 6-APA by BlaM (red line in Figure 5A). At 50 $\mu \mathrm{g} / \mathrm{ml}$ of 6-APA, the discrepancy between un-induced (green line in Figure 5B) and induced populations (red line in Figure 5B) was even greater. For the population without IPTG induction, the inflection point of population growth was slightly shifted to shorter time due to higher stress compared to $25 \mu \mathrm{g} / \mathrm{ml}$ 6-APA. At $100 \mu \mathrm{g} / \mathrm{ml}$ of 6-APA, however, the discrepancy between the two populations appeared to decrease (Figure 5C, Supporting Video S2). This reduced discrepancy was likely 
due to the inability of released BlaM (from the IPTG-induced population) to effectively degrade the high concentration of antibiotic. The effect of this higher antibiotic concentration was also reflected by the inflection point of population growth without IPTG, which shifted to $250 \mathrm{~min}$ (green line in Figure 5C). At $400 \mu \mathrm{g} / \mathrm{ml}$ of 6-APA, the PAD population could not survive with or without IPTG induction (Figure S5). In contrast, NPD cells could still grow to high density even without IPTG rescuing due to the higher tolerance.

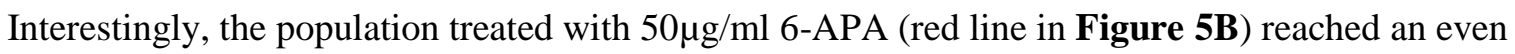
higher final density than that with $25 \mu \mathrm{g} / \mathrm{ml}$ 6-APA (red line in Figure 5A), when both of them were rescued by $1 \mathrm{mM}$ IPTG. We speculate that $50 \mu \mathrm{g} / \mathrm{ml} 6$-APA could approximate to the optimal death rate [14] that correlates to the production rate of BlaM. That is, the lysis of cells and production of BlaM are better balanced to degrade the antibiotic and survive the whole population. However, for $25 \mu \mathrm{g} / \mathrm{ml}$ 6-APA, the cells were lysed more slowly due to the lower concentration of local E lysis protein that led to longer time to release BlaM. In contrast, for $100 \mu \mathrm{g} / \mathrm{ml} 6-\mathrm{APA}$, the death rate was faster than the production rate of $\beta$-lactamase, leading to insufficient degradation of 6-APA.

To further demonstrate the manipulability of the droplet environment, we modulated the concentration of IPTG that could regulate the production of BlaM. In this case, we injected varying concentrations of IPTG but the same concentration of 6-APA $(50 \mu \mathrm{g} / \mathrm{ml})$ into the droplets containing the PAD strain. When the final concentration was estimated as $0.02 \mathrm{mM}$, IPTG could not rescue the population due to insufficient induction of BlaM (Figure S6). At higher concentrations of IPTG $(0.1 \mathrm{mM}$ and $0.5 \mathrm{mM})$, the PAD population in droplets could indeed be better rescued and grew to higher final cell densities. No drastic difference was detected from these two concentrations, which demonstrated that $0.1 \mathrm{mM}$ IPTG might be sufficient in droplets to rescue the population with $50 \mu \mathrm{g} / \mathrm{ml} 6$-APA. 


\section{Discussion}

Microfluidics can greatly facilitate culturing and measuring of bacterial populations [43], thanks to the drastically reduced use of reagents and improved mass transfer [2, 44]. However, the design of conventional microfluidic channels can be limited by the Taylor dispersion [45], creation of isolated microenvironment to prevent cross-contamination or high-throughput outcomes for statistical analysis $[46,47]$. In contrast, droplet technology is advantageous in terms of isolated environment, fast production and high throughput [17]. Indeed, several studies have demonstrated the use of droplets in culturing and monitoring bacterial population dynamics, using single- [21] or double emulsions [16]. However, a major challenge is the difficulty in manipulating the chemical environment of individual droplets to achieve dynamic control of cellular behaviors. This ability is critically important to probe bacterial dynamics in response to different environment cues.

For double emulsions, this can be done by relying on passive diffusion of a chemical from the outer water phase, through the oil phase, and into inner water core [16]. However, the efficiency of this process is limited by the partition constant of the chemical between the water and oil phases. The transport of chemicals with low partition constant is highly inefficient. A more efficient alternative is to directly inject a chemical of interest, as demonstrated by our results. Such injection would be difficult to achieve in double-emulsion droplets, due to the need to coordinate the injection phase and the droplet generation phase and break two layers of surfactant layers. Also, our electrode-free injection technique is more cost-effective than those using physical electrodes: no costly instrument (e.g. thin film deposition system for electrode layer), metal materials (e.g. $\mathrm{Cr}$, Ti or $\mathrm{Au}$ ), or extensive labor (e.g. fabrication of electrode patterns) is required. 
Furthermore, our work developed another technique that enabled long-term monitoring of population dynamics in trapped droplets, by limiting the evaporation of the oil phase. By using this technique, we were able to culture the bacteria for at least $240 \mathrm{hrs}$. To what we know, this is the longest bacterial culturing and monitoring ever achieved by using droplets.

Overall, both engineered and natural bacteria in droplets generated population dynamics that were consistent with the expected system behaviors in each case. This consistency indicates the versatility of our system to analyze diverse bacterial dynamics. However, these results exhibited some quantitative differences from those generated from bulk cultures. For example, the ePop circuit in this work exhibited fewer oscillation cycles [31] and a different concentration of Strep was observed to generate IE [35]. These differences most likely result from the different growth environments. For instance, given the confinement in droplets, oxygen diffusion is limited by outer oil phase and immersion water in the sealed petri dish. As such, the growth environment in the droplet is more anaerobic than in bulk cultures. Also, due to the small size of droplets, the initial cell number in each droplet can be highly variable, which could have contributed to the variability in the growth dynamics between droplets. Yet, the ability to generate such heterogeneity, on the other hand, is critical for quantifying the evolutionary dynamics of cooperation $[10,12,13]$, as well as the demographic stochasticity in predator-prey dynamics [48]. Altogether, our work demonstrated the feasibility of using liquid droplets to control and quantify dynamics of small bacterial populations, which has implications for the analysis of population dynamics of bacteria or other microbes [49] in diverse contexts. 


\section{Acknowledgement}

We thank Yu Tanouchi for useful comments on the manuscript and the Shared Materials and Instrumentation Facility at Duke University for assisting with fabrication of our microfluidic devices. This work was partially supported by the National Science Foundation (LY, CBET0953202), the National Institutes of Health (R01GM098642 (LY), R01GM110494 (LY \& KL)), the Army Research Office (LY), a DuPont Young Professorship (LY), and a David and Lucile Packard Fellowship (LY). 


\section{References}

[1] Balazsi G, van Oudenaarden A, Collins JJ. Cellular Decision Making and Biological Noise: From Microbes to Mammals. Cell. 2011;144:910-25.

[2] Balagadde FK, You L, Hansen CL, Arnold FH, Quake SR. Long-term monitoring of bacteria undergoing programmed population control in a microchemostat. Science. 2005;309:137-40.

[3] Tan C, Smith RP, Tsai MC, Schwartz R, You L. Phenotypic signatures arising from unbalanced bacterial growth. Plos Comput Biol. 2014;10:e1003751.

[4] Thomas CM, Nielsen KM. Mechanisms of, and barriers to, horizontal gene transfer between bacteria. Nat Rev Microbiol. 2005;3:711-21.

[5] Riccione KA, Smith RP, Lee AJ, You LC. A Synthetic Biology Approach to

Understanding Cellular Information Processing. Acs Synth Biol. 2012;1:389-402.

[6] Kotula JW, Kerns SJ, Shaket LA, Siraj L, Collins JJ, Way JC, et al. Programmable

bacteria detect and record an environmental signal in the mammalian gut. P Natl

Acad Sci USA. 2014;111:4838-43.

[7] Balagadde FK, Song H, Ozaki J, Collins CH, Barnet M, Arnold FH, et al. A synthetic Escherichia coli predator-prey ecosystem. Mol Syst Biol. 2008;4.

[8] West SA, Griffin AS, Gardner A, Diggle SP. Social evolution theory for microorganisms. Nat Rev Microbiol. 2006;4:597-607.

[9] Velenich A, Gore J. Synthetic approaches to understanding biological constraints. Current opinion in chemical biology. 2012;16:323-8.

[10] Xavier JB. Social interaction in synthetic and natural microbial communities. Mol Syst Biol. 2011;7:483.

[11] Pai A, Tanouchi Y, You LC. Optimality and robustness in quorum sensing (QS)mediated regulation of a costly public good enzyme. P Natl Acad Sci USA.

2012;109:19810-5.

[12] Chuang JS, Rivoire 0, Leibler S. Simpson's Paradox in a Synthetic Microbial System. Science. 2009;323:272-5.

[13] Chuang JS, Rivoire 0, Leibler S. Cooperation and Hamilton's rule in a simple synthetic microbial system. Mol Syst Biol. 2010;6:398.

[14] Tanouchi Y, Pai A, Buchler NE, You LC. Programming stress-induced altruistic death in engineered bacteria. Mol Syst Biol. 2012;8.

[15] Clausell-Tormos J, Lieber D, Baret JC, El-Harrak A, Miller OJ, Frenz L, et al. Droplet-based microfluidic platforms for the encapsulation and screening of mammalian cells and multicellular organisms (vol 15, pg 427, 2008). Chem Biol. 2008;15:875-.

[16] Zhang Y, Ho YP, Chiu YL, Chan HF, Chlebina B, Schuhmann T, et al. A programmable microenvironment for cellular studies via microfluidics-generated double emulsions. Biomaterials. 2013;34:4564-72.

[17] Teh SY, Lin R, Hung LH, Lee AP. Droplet microfluidics. Lab Chip. 2008;8:198220.

[18] Gu H, Duits MHG, Mugele F. Droplets Formation and Merging in Two-Phase Flow Microfluidics. Int J Mol Sci. 2011;12:2572-97. 
[19] Jakiela S, Kaminski TS, Cybulski O, Weibel DB, Garstecki P. Bacterial Growth and Adaptation in Microdroplet Chemostats. Angew Chem Int Edit. 2013;52:8908-11. [20] Weitz M, Muckl A, Kapsner K, Berg R, Meyer A, Simmel FC. Communication and Computation by Bacteria Compartmentalized within Microemulsion Droplets. J Am Chem Soc. 2014;136:72-5.

[21] Boedicker JQ, Li L, Kline TR, Ismagilov RF. Detecting bacteria and determining their susceptibility to antibiotics by stochastic confinement in nanoliter droplets using plug-based microfluidics. Lab Chip. 2008;8:1265-72.

[22] Mazutis L, Gilbert J, Ung WL, Weitz DA, Griffiths AD, Heyman JA. Single-cell analysis and sorting using droplet-based microfluidics. Nat Protoc. 2013;8:870-91. [23] Park J, Kerner A, Burns MA, Lin XXN. Microdroplet-Enabled Highly Parallel CoCultivation of Microbial Communities. PloS one. 2011;6.

[24] Brouzes E, Medkova M, Savenelli N, Marran D, Twardowski M, Hutchison JB, et al. Droplet microfluidic technology for single-cell high-throughput screening. Proc Natl Acad Sci U S A. 2009;106:14195-200. [25] O'Donovan B, Eastburn DJ, Abate AR. Electrode-free picoinjection of microfluidic drops. Lab Chip. 2012;12:4029-32.

[26] Duffy DC, McDonald JC, Schueller OJA, Whitesides GM. Rapid prototyping of microfluidic systems in poly(dimethylsiloxane). Anal Chem. 1998;70:4974-84.

[27] Baret JC. Surfactants in droplet-based microfluidics. Lab Chip. 2012;12:422-33. [28] Bringer MR, Gerdts CJ, Song H, Tice JD, Ismagilov RF. Microfluidic systems for chemical kinetics that rely on chaotic mixing in droplets. Philos T Roy Soc A. 2004;362:1087-104.

[29] Courtois F, Olguin LF, Whyte G, Bratton D, Huck WT, Abell C, et al. An integrated device for monitoring time-dependent in vitro expression from single genes in picolitre droplets. Chembiochem : a European journal of chemical biology. 2008;9:439-46.

[30] Huebner A, Bratton D, Whyte G, Yang M, deMello AJ, Abell C, et al. Static microdroplet arrays: a microfluidic device for droplet trapping, incubation and release for enzymatic and cell-based assays. Lab Chip. 2009;9:692-8.

[31] Marguet P, Tanouchi Y, Spitz E, Smith C, You L. Oscillations by minimal bacterial suicide circuits reveal hidden facets of host-circuit physiology. PloS one. 2010;5:e11909.

[32] Henrich B, Lubitz W, Plapp R. Lysis of Escherichia coli by induction of cloned phi X174 genes. Molecular \& general genetics : MGG. 1982;185:493-7.

[33] Young KD, Young R. Lytic action of cloned phi X174 gene E. Journal of virology. 1982;44:993-1002.

[34] Brook I. Inoculum effect. Reviews of infectious diseases. 1989;11:361-8. [35] Tan C, Smith RP, Srimani JK, Riccione KA, Prasada S, Kuehn M, et al. The inoculum effect and band-pass bacterial response to periodic antibiotic treatment. Mol Syst Biol. 2012;8:617.

[36] Meredith HR, Srimani JK, Lee AJ, Lopatkin AJ, You L. Collective antibiotic tolerance: mechanisms, dynamics and intervention. Nat Chem Biol. 2015;11:182-8. [37] Soriano F, Ponte C, Santamaria M, Jimenezarriero M. Relevance of the Inoculum Effect of Antibiotics in the Outcome of Experimental Infections Caused by Escherichia-Coli. J Antimicrob Chemoth. 1990;25:621-7. 
[38] Raymon LP. COMLEX Level 1 Pharmacology Lecture Notes. Miami, FL: Kaplan, Inc. 2011;p. 181. CM4024K.

[39] Goff SA, Goldberg AL. Production of abnormal proteins in E. coli stimulates transcription of lon and other heat shock genes. Cell. 1985;41:587-95.

[40] Vanbogelen RA, Neidhardt FC. Ribosomes as Sensors of Heat and Cold Shock in Escherichia-Coli. P Natl Acad Sci USA. 1990;87:5589-93.

[41] Sykes MT, Shajani Z, Sperling E, Beck AH, Williamson JR. Quantitative Proteomic Analysis of Ribosome Assembly and Turnover In Vivo. Journal of molecular biology. 2010;403:331-45.

[42] Jacobs C, Frere JM, Normark S. Cytosolic intermediates for cell wall biosynthesis and degradation control inducible beta-lactam resistance in gram-negative bacteria. Cell. 1997;88:823-32.

[43] Breslauer DN, Lee PJ, Lee LP. Microfluidics-based systems biology. Mol Biosyst. 2006;2:97-112.

[44] Jensen KF. Microchemical systems: Status, challenges, and opportunities. Aiche J. 1999;45:2051-4.

[45] Wunderlich B, Nettels D, Schuler B. Taylor dispersion and the position-to-time conversion in microfluidic mixing devices. Lab Chip. 2014;14:219-28.

[46] deMello AJ. Control and detection of chemical reactions in microfluidic systems. Nature. 2006;442:394-402.

[47] Solvas XCI, deMello A. Droplet microfluidics: recent developments and future applications. Chem Commun. 2011;47:1936-42.

[48] McKane AJ, Newman TJ. Predator-prey cycles from resonant amplification of demographic stochasticity. Phys Rev Lett. 2005;94.

[49] Nevozhay D, Adams RM, Van Itallie E, Bennett MR, Balazsi G. Mapping the Environmental Fitness Landscape of a Synthetic Gene Circuit. Plos Comput Biol. 2012;8. 


\section{Figures}

A

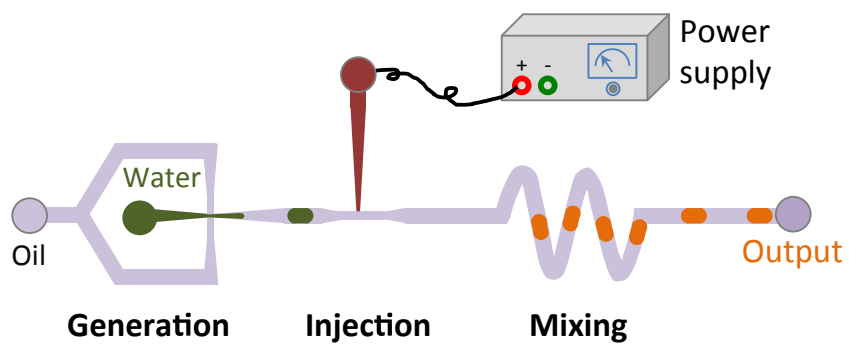

B Surfactant molecules
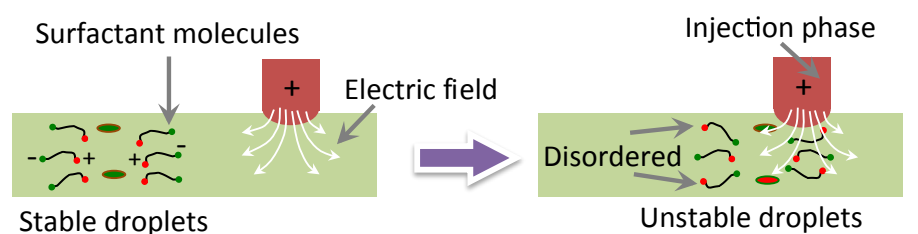

C
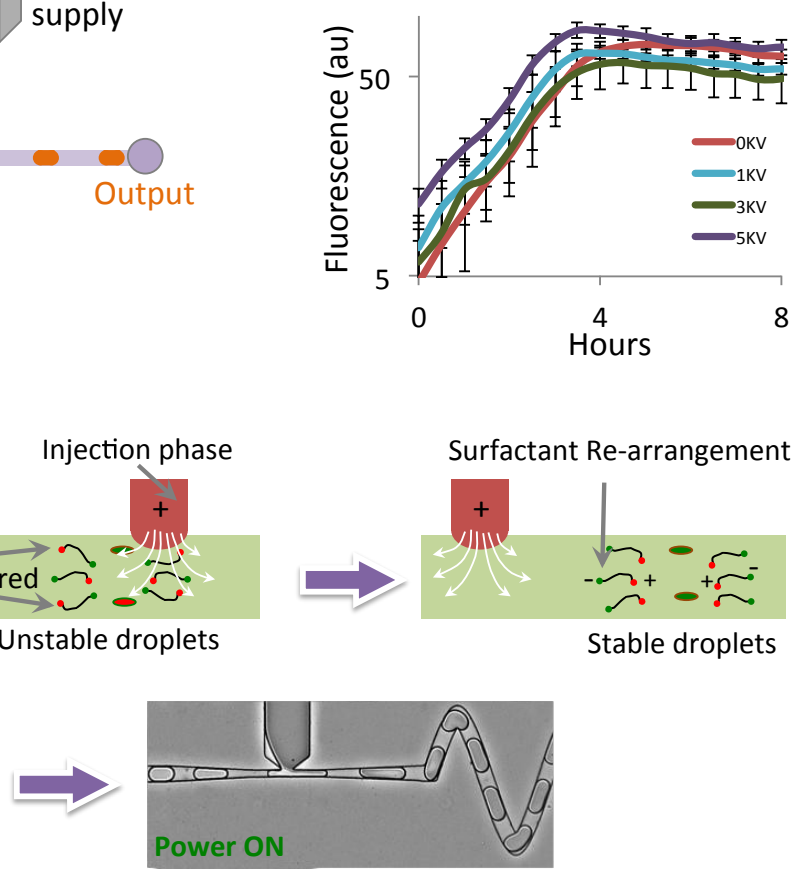

Figure1. A microfluidic platform for droplet production and injection.

(A) Schematic of droplet injection chip. The microfluidic device consists of droplet production, injection and mixing units. The droplet injection is triggered by DC power supply.

(B) Mechanism of droplet injection. A layer of surfactant molecules stabilizes the droplets after production. An electric field disrupts this protecting layer when power is turned $\mathrm{ON}$, and the injection phase is injected into the original droplets when they move across the orifice. Real-time images depict the difference between power OFF and ON. Orange arrows indicate the individual droplets from injection phase without being injected.

(C) Effects of electric field on cell survival. The droplets were injected with M9 media under varied voltage values. Mean fluorescence intensity of each droplet (log scale) was quantified over time. Error bars correspond to \pm 1 standard deviation of averaged fluorescence intensity of all droplets $(n>20)$. 

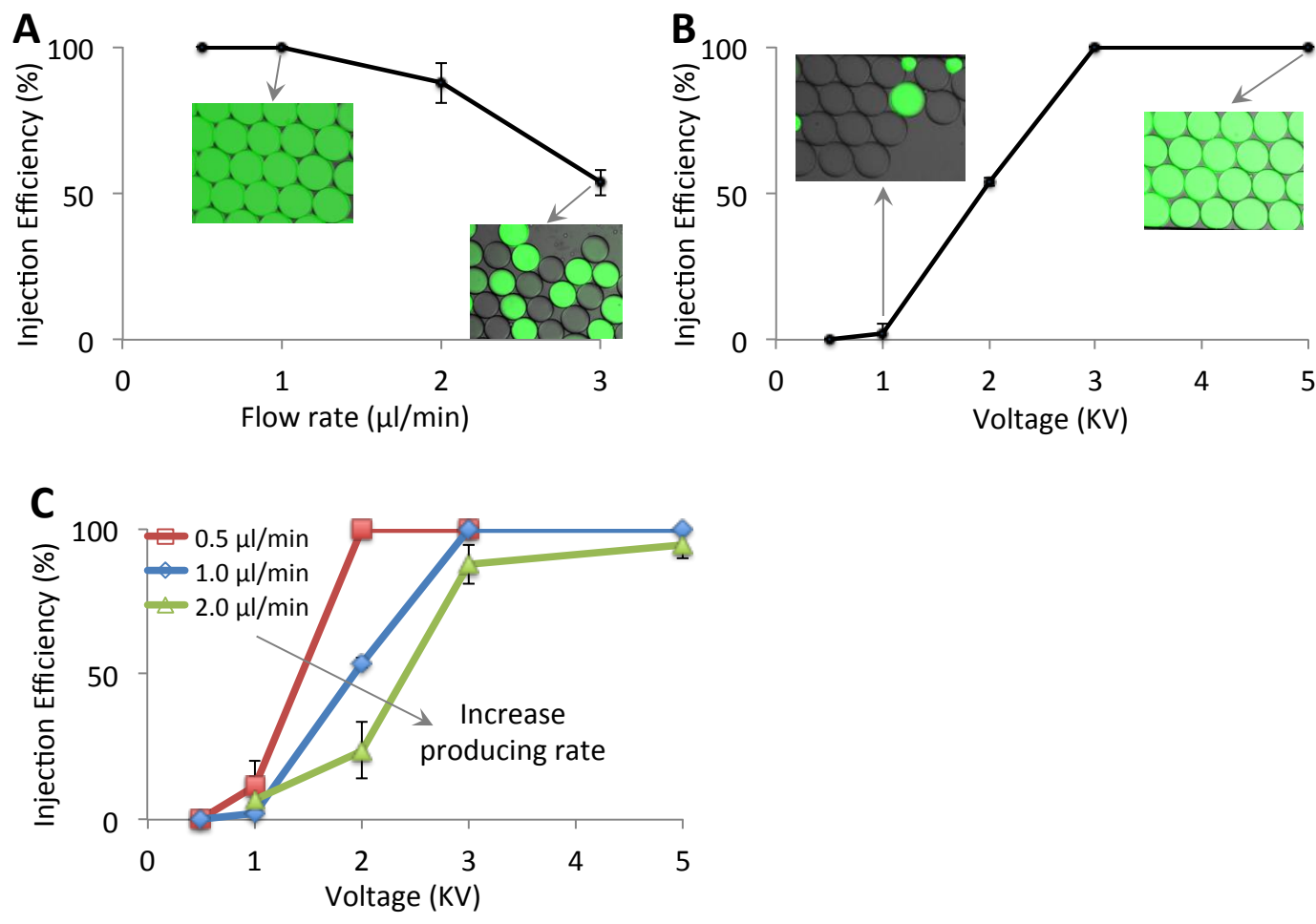

\section{Figure 2. Tunability of droplet injection technology.}

(A) Tunable injection efficiency by production rate of droplets. Voltage was set as $3 \mathrm{KV}$ to trigger all injections, and flow rate of injection phase was $0.1 \mu \mathrm{l} / \mathrm{min}$. Flow rates of oil and water phase were set as the same and varied from $0.5 \mu \mathrm{l} / \mathrm{min}$ to $3 \mu \mathrm{l} / \mathrm{min}$. $1.0 \mu \mathrm{l} / \mathrm{min}$ was chosen for subsequent experiments regarding the injection efficiency and production rate of droplets.

(B) Tunable injection efficiency by voltage. Flow rates of oil and water phase were both set as $1.0 \mu \mathrm{l} / \mathrm{min}$, and injection phase was $0.1 \mu \mathrm{l} / \mathrm{min}$. Voltage was varied from $0 \mathrm{KV}$ to $5 \mathrm{KV}$ to trigger the injection. Regarding the injection efficiency and homogeneity of droplet size, $3.0 \mathrm{KV}$ was chosen for subsequent experiments.

(C) Correlation between injection efficiency and voltage value when production rate of droplets was varied. Flow rates of both oil and water phase were the same and varied from $0.5 \mu \mathrm{l} / \mathrm{min}$ to $2 \mu \mathrm{l} / \mathrm{min}$, and voltage from $0 \mathrm{KV}$ to $5 \mathrm{KV}$. Dash line denoted the threshold of voltage to obtain sufficient efficiency for the application of droplet injection. Error bars denoted \pm 1 standard deviation from multiple detecting positions (see details in $\mathrm{SI})$. 

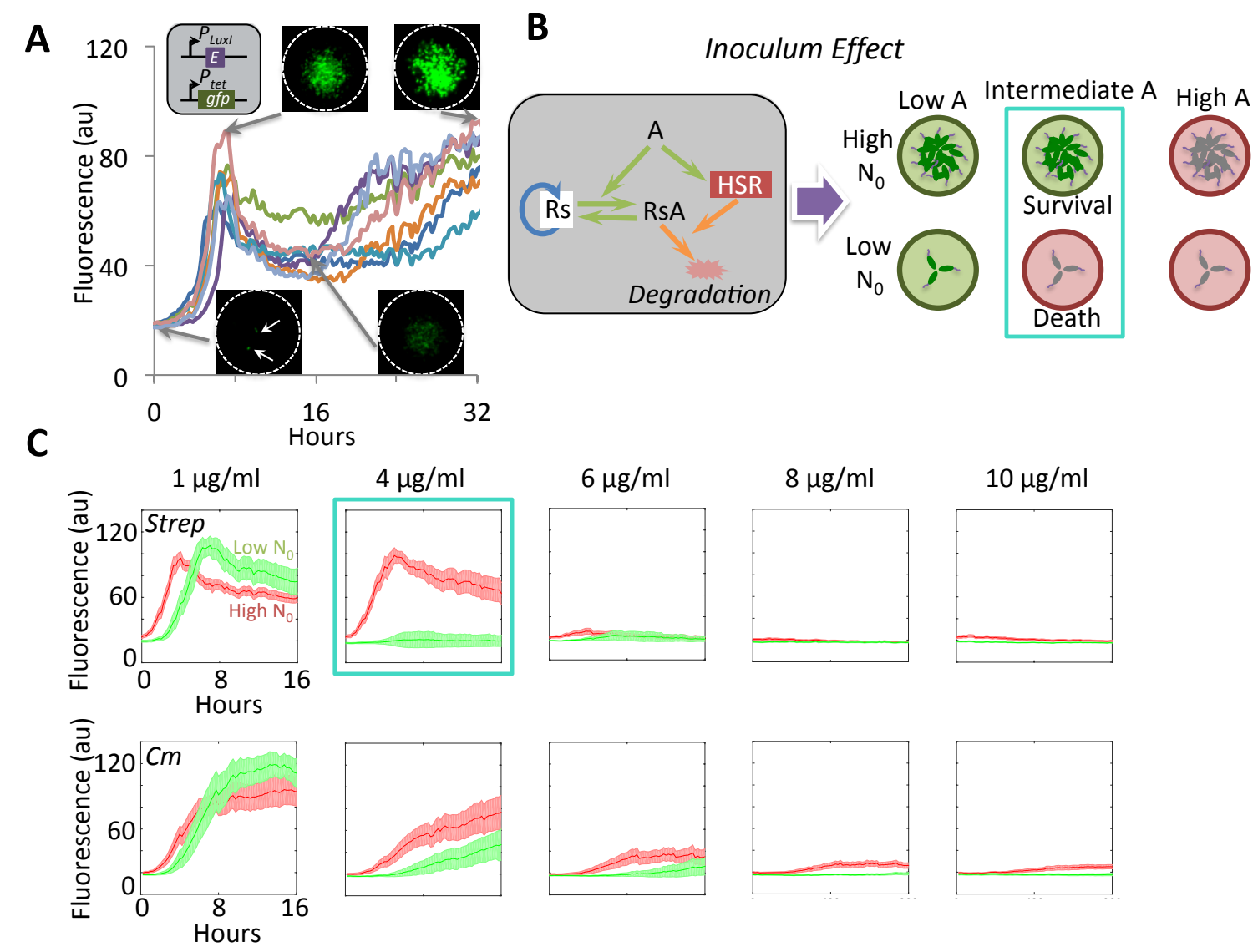

Figure 3. Droplets used for quantification of bacterial population dynamics.

(A) Quantification of multiple droplets with population collapse and recovery with an ePop circuit. Each line represents one subpopulation in droplet started with low cell density (1 5 cells per droplet). Images are the representative time points of ePop oscillation. The top left inset represents schematic of the ePop circuit, GFP reporter works as an indicator of cell viability.

(B) Schematic of inoculum effect (IE). Antibiotic (A) targets ribosome (Rs) to inhibit its accumulation and mislead protein synthesis. Concurrently, A causes heat shock response (HSR) to further destroy the ribosome feedback (Left). At low concentration, A cannot efficiently inhibit Rs, and population with both high and low initial densities (N0) will initiate growth. Conversely, high concentration of A effectively inhibits growth for both populations. Importantly, IE can only happen at intermediate concentration of A (Right). (C) Investigation of IE in droplets. The bacterial strain with GFP reporter were encapsulated in droplets with varied concentrations of antibiotics. Streptomycin (Strep) 
concentrations below $4 \mu \mathrm{g} / \mathrm{ml}$ were insufficient to inhibit growth of population with both low (green) and high (red) initial density (N0), while concentrations greater than $4 \mu \mathrm{g} / \mathrm{ml}$ effectively prevented growth of both conditions. IE was only observed when Step was $4 \mu \mathrm{g} / \mathrm{ml}$. In comparison, chloramphenicol $(\mathrm{Cm})$ did not generate IE within the same range of concentration. The curves indicate mean fluorescence intensity of sampled droplets $(\mathrm{n}>20)$ versus time, and shades refer to the \pm 1 standard deviation. 
A

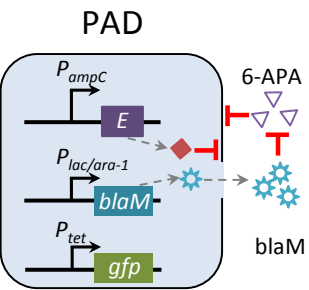

B

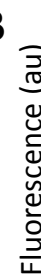

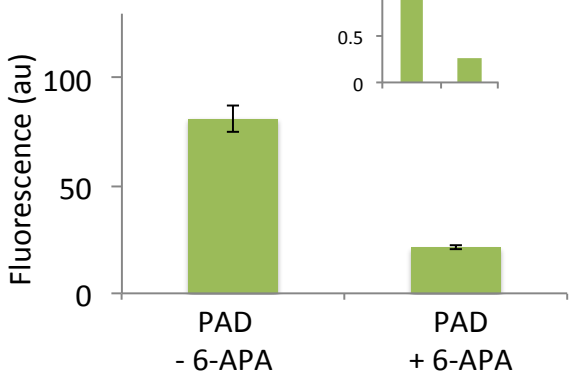

C

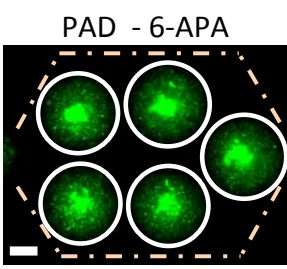

PAD + 6-APA

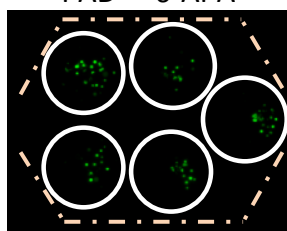

NPD
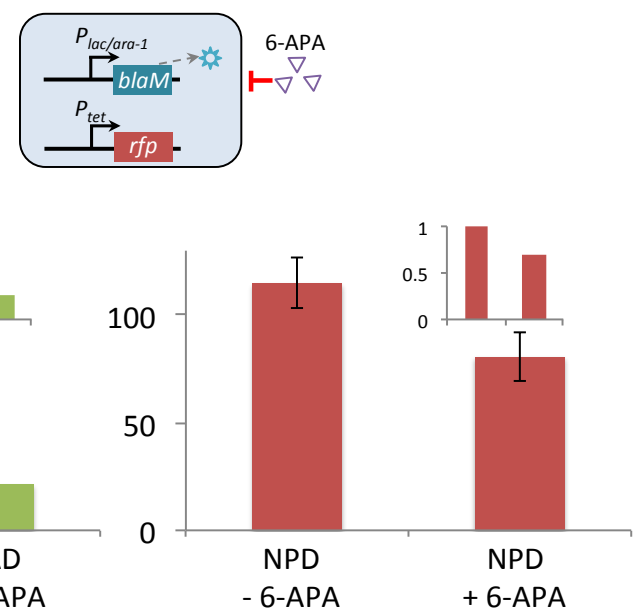

NPD - 6-APA

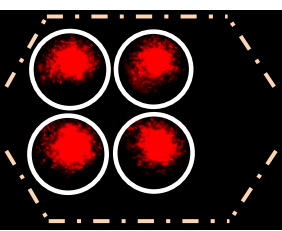

NPD + 6-APA

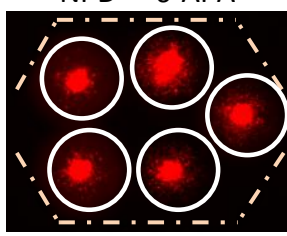

Figure 4. Droplet injection for control of population dynamics.

(A) Schematics of PAD and NPD circuits. PAD consists of two modules, E lysis module and $\beta$-lactamase (BlaM) module. 6-APA partially breaks down cell murein, and generates the intermediate that can induce the synthesis of E protein. E protein further lyses the cell to release BlaM into the environment, which can then degrade 6-APA. BlaM is placed under an inducible IPTG promoter; constitutively expressed GFP is used as a reporter for cell viability. For control, the E lysis module is removed in the non-programmed altruistic death (NPD) circuit, and RFP is used as reporter.

(B) Inhibition of PAD growth by 6-APA. PAD cells in the droplets that were injected with M9 (PAD - 6-APA) grew to high density after 24 hours. In contrast, growth of PAD strain was fully inhibited in droplets when $400 \mu \mathrm{g} / \mathrm{ml} 6$-APA was injected (PAD + 6APA). This inhibition was not significant for NPD cells due to the deletion of E lysis module (NPD + 6-APA) compared to those without 6- APA (NPD - 6-APA). The insets demonstrated the normalized growth for the conditions with or without 6-APA treatment. Error bars represent \pm 1 standard deviation of average at 24 th hour of culture $(n>20)$.

(C) Representative images of (B). The scale bar represents $25 \mu \mathrm{m}$. 

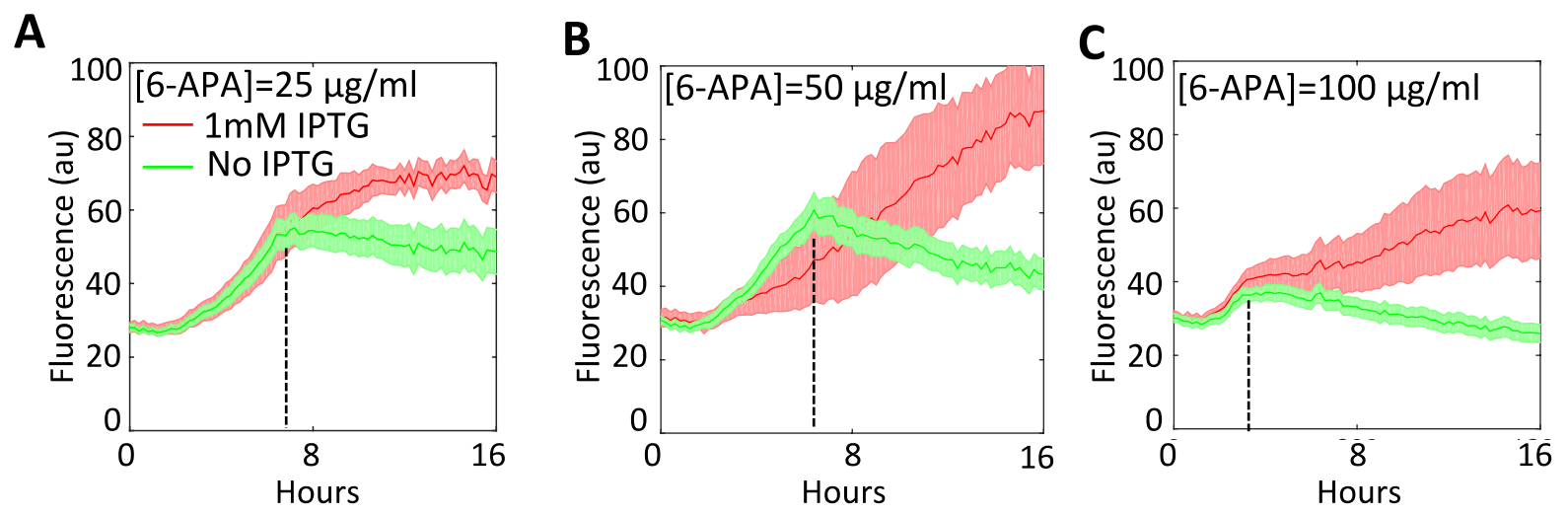

Figure 5. Regulation of PAD by injecting different concentrations of 6-APA into droplets.

(A) $25 \mu \mathrm{g} / \mathrm{ml}$ (B) $50 \mu \mathrm{g} / \mathrm{ml}$ and (C) $100 \mu \mathrm{g} / \mathrm{ml} 6$-APA was injected into droplets with (red) and without (green) $1 \mathrm{mM}$ IPTG rescuing. Black dash lines indicate inflection points when the populations (green) stop growing. 